\title{
ON THE ENERGY RELATIONS OF SOLUTE AND SOLVENT
}

BY M. M. GARVER.

In a former paper ${ }^{1}$ attention was called to the fact that an expression for the maximum work done during the dilution of a dilute solution was independent of any force action on the part of the dissolved substance. Further study of the experimental evidence afforded by osmotic phenomena serves but to strengthen my conviction of the general soundness of the views therein presented, except as to minor details; in some respects those are open to modifications. The main fact, however, that the phenomena of osmosis all arise from and are most simply explained in terms of the activity of the solvent instead of the solute, seems beyond doubt when the deductions from such a view are considered as a whole. The object of the present paper is to show how a few of the many experimental facts available may be interpreted in the light of the proof that the dissolved substance takes no active part in the production of the maximum work done during dilution. And if the part it plays in the production of work be purely a passive one, may not the same passivity be traced throughout the whole range of the phenomena connected with dilute solutions? The question seems one worthy of investigation in view of the important part taken by osmotic pressure in modern science. But at the very outset we are met by the fact that osmotic pressure has been used as a measure of the work done in separating the constituents of a solution and has given perfectly consistent results from that point of view. The statement, then (with proof which cannot be doubted), that the dissolved substance plays only a passive part in the production of work would make osmotic pressure dependent on the activity of the solvent. Practically all of the text-books speak of osmotic pressure as a pressure exerted by the dissolved substance

${ }^{1}$ Jour. Phys. Chem., r3, 679 (rgog). 
and yet use it as a measure of work done on or by the solution. Here is an evident conflict that requires clearing up. Either the alleged "proof" is false or a reinterpretation of osmotic pressure is demanded. If no defect can be found in the proof then a re-examination of the experimental evidence is absolutely necessary.

Evidently the first thing in order is a re-examination of the proof that the maximum work done during the dilution of a dilute solution is independent of any activity of the solute. However, the proof may readily be verified in different ways. The simplest and most satisfactory method will probably be afforded by evaluating the integral regarded as proof and showing that it leads directly to accepted canonic forms.

In the former paper (op. cit.) an expression for the maximum work was obtained from $d \mathrm{~W}=g h d m$ which gives $\mathrm{W}=\rho g \int_{h_{1}}^{h_{9}} h d v$ where $g$ is the attraction of gravity, $\rho$ is the density of the solvent, $v$ its volume and $h$ a variable height. Before this can be evaluated it will be necessary to find the functional relation between $h$ and $v$ subject to the condition that the work between the limits is a maximum. Using the same device described in detail in the former paper, we may at any point regard $h$ as constant and gradually change the volume by adding solute-so slowly that the equilibrium will not be disturbed, the vapor condensing will keep the concentration constant. Now the integral will take the form

$$
\mathrm{W}=\rho g h \int_{v_{1}}^{v_{2}} d v .
$$

This is still a maximum, but gives the entire work due to dissolving (instead of diluting) an unknown, but definite mass of solute. It will serve to give the functional relation between $h$ and $v$ when the work is a maximum for a definite mass of solute, $h$ arbitrary and the volume varied between 
$v_{1}$ and $v_{2}$. We have then, $\mathrm{W}=\rho g h\left(v_{2}-v_{1}\right)$, which becomes $\rho g h v$ when $v_{1}$ is negligibly small. (We may start with as small a volume as we please.) Since the work is a maximum for that amount of solute, we may write

$$
\mathrm{W}^{\prime}=\rho g h v=\text { const. }
$$

Hence we see that $h$ varies inversely as $v$, therefore $h d v=$ $-v d h$ and $v=\mathrm{W}^{\prime} / \rho g h$. Therefore making the substitutions and integrating we have

$$
\mathrm{W}==\mathrm{W}^{\prime} \int_{h_{1}}^{h_{2}} \frac{d h}{h}=\rho g h v \log _{\varepsilon} \frac{h_{2}}{h_{1} .}
$$

If for $\rho g h$ we write $p$, and for $\frac{h_{2}}{h_{1}}$ write its equal, $v_{1} / v_{2}$, we have $\mathrm{W}=p v \log \frac{v_{1}}{v_{2}}$ the accepted canonic form, for the maximum work obtainable through diluting a solution between the limits above indicated, $v_{1}$ and $v_{2}$.

Except as a verification we are not just now interested in the canonic form. Let us examine and analyze our results. In the first place it may be noted from the selected order of the limits, since $h_{1}>h_{2}$, that work done on the solution is positive; work done by the solution, negative.

It is well also to note that the text-books also give the osmotic pressure $\mathrm{P}=\rho g h$ or an equivalent expression; but this result is obtained only by assuming the validity of the application of van't Hoff's form of the general gas equation.

From the fact that $p v$, or $\mathrm{PV}$, represents the maximum work, the maximum work must be proportional to $\mathrm{P}$; and since the maximum, or limiting, value of $P$ was always understood and used it would not fail to give consistent results. But from the present method of deriving the expression for the maximum work there is as yet no evidence to show that $\rho g h$ represents a pressure beyond the fact that in mechanics $\rho g h$ represents the pressure per unit area of a fluid column. In the van't Hoff equation there was always an ambiguity 
attached to the term "volume." The present method leaves no doubt as to the volume represented but leaves $\rho g h$ ambiguous. What does it mean? Since $\rho v=m$, the full expression $\rho g h v$ may be written $\mathrm{W}=m g h$, the work in dynecentimeters, or foot poundals, or work expressed in terms of displacement against resistance. Since work, energy, can always be expressed in terms of $F s$ or $\mathrm{P} \mathrm{V}$ and must be expressed in some such way in order to be intelligible, or comparable, we may reason that since $\mathrm{W}$ and $v$ are definite physical quantities, $\rho g h$ must represent a definite physical pressure. It is numerically equal to what has been called osmotic pressure and is a physical pressure because it represents the ratio between work and volume. Let us endeavor to interpret it without introducing any hypotheses as to the constitution of matter or making any assumptions not warranted by experimental and dynamic considerations.

It was noted above that the maximum work obtainable by dissolving a solid in a liquid under certain specified conditions, was just sufficient to lift the entire mass of the pure solvent to a height which would serve to equilibrate the vapor pressures of the solution and pure solvent at the point of contact. This is important and must be borne in mind in connection with the development of the subject. In the ideal experiment detailed in the former paper, the vapor lifted itself just that height. This fact, in connection with the other fact, that no evidence of activity on the part of the solute was traceable, suggests an assumption, purely dynamic, which will be justified if it leads to results experimentally verifiable.

Since in the derivation of the maximum work we found that the energy came from the solvent, we might assume that all the energy taking part in the work is proportional to the amount of solvent in the solution. This is evidently the case from $p v=\mathrm{W}$ and in such cases as we have been considering where the work is done by rising vapor, but is not so evident in the osmotic cell or during the passage of 
the solvent through a semi-permeable wall. Now Planck ${ }^{1}$ shows that in the case of gases the pressure of the gas that does not pass through the semi-permeable wall has no influence, whatever its pressure, on the equilibrating pressures of the gas that can pass through. Therefore, whatever the pressure of the dissolved substance, we may neglect it and state the conditions of equilibrium as though it were absent. This of course is assuming the above law to hold for liquids. Let us consider the osmotic cell with an open manometer tube just for the sake of visualizing the operation. We may also imagine it under a cover with the air exhausted.

The assumption that all the energy engaged in doing work comes from the solvent is equivalent to assuming that the ratio of the energy per unit volumes of the liquid to vapor is the same for both solution and pure solvent. The condition of equilibrium then, since the liquid can pass through the semi-permeable wall, will be given by assuming that since energies are proportional to pressures,

$$
\frac{p_{1}}{\mathrm{P}_{1}}=\frac{p_{2}}{\mathrm{P}_{2}} \text { or } \frac{p_{1}}{p_{2}}=\frac{\mathrm{P}_{1}}{\mathrm{P}_{2}}
$$

where $p_{1}$ is the vapor pressure of the pure solvent, $P_{1}$ the inwardly directed pressure of the pure liquid solvent, $p_{2}$ the vapor pressure of the solution and $\mathrm{P}_{2}$ the outwardly directed pressure of the liquid solution,-the effect at any point of contact being assumed proportional to amount of material acting at that point or element of area. We may write these ratios

$$
\frac{p_{1}-p_{2}}{p_{1}}=\frac{\mathrm{P}_{1}-\mathrm{P}_{2}}{\mathrm{P}_{1}}
$$

which will be constant for a given concentration but will vary with the concentration. Therefore $p_{1}-p_{2}=\mathrm{C} p_{1}$. $\mathrm{P}_{1}-\mathrm{P}_{2}=\mathrm{CP}_{1}$. But $p_{1}-p_{2}$ being a difference in vapor pressures, and $\mathrm{P}_{1}-\mathrm{P}_{2}$ being a difference in liquid pressures due to solvent only, we may write

$$
p_{1}-p_{2}=\rho^{\prime} g h \text { and } \mathrm{P}_{1}-\mathrm{P}_{2}=\rho g h \text { (osmotic pressure) }
$$

\footnotetext{
${ }_{1}^{1}$ Treatise on Thermodynamics. Translated by Ogg, p. 2 Io.
} 
where $\rho^{\prime}$ is the average density of the vapor of the pure solvent and $\rho$ is the density of the liquid solvent and $h$ is the height necessary to equilibrate the pressures whether liquid or vapor. Eliminating $g h$ we have

$$
\frac{p_{1}-p_{2}}{\rho^{\prime}}=\frac{P_{1}-P_{2}}{\rho}
$$

or regarding the differences as very small and integrating, from $o$ to $p$, or $\mathrm{P}$, we get $\frac{d p}{\rho^{\prime}}=\frac{d \mathrm{P}}{\rho} \cdot \frac{p}{\rho^{\prime}}=\frac{\mathrm{P}}{\rho}$ or, $p v=\mathrm{PV}=\mathrm{RT}$ by the gas laws, on substituting volumes to replace densities.

It may be noticed that the key to the above deduction turns on the assumption that the two opposed pressures are proportional to the amount of material of the solvent, whether liquid or vapor, acting in opposite directions on opposite sides of the areas where they are to be equilibrated. This is assumed to apply to the non-homogeneous layer between the vapors as well as to the semi-permeable wall between the liquids. The assumption is seen to be the simplest possible required to explain the dynamic facts, and was suggested by the height of the liquid column representing the maximum work. Why should equilibrium be established at just that height? Evidently because the energy concerned was exactly proportional to the quantity of substance available to produce the observed action.

It should be observed that no molecular or atomic or corpuscular constitution of matter was assumed, but purely dynamic relations. The kinetic theory assures us that the activity of the molecules of a substance depends upon the absolute temperature alone and is independent of the liquid or vapor state. The result of the above deduction really depends on this being true; the result may be taken as a corroboration.

There is an entirely different method of deducing the value of $\rho g h$ considered as a pressure, and, in some respects, is preferable since the deduction does not depend upon osmosis but does depend upon the possibility of separating by filtration the constituents of a solution. In fact the 
equilibrium method above given just occurred to me while writing; the filtration method was originally in mind and accounts for the phraseology of the first part of the paper.

On the assumption that we are dealing only with what might be called a mechanical, or physical, solution of a solid in a liquid unaccompanied by chemical action by which heat is liberated, $p v=\mathrm{W}$ represents the total mechanical work, or free energy, available by completely dissolving a given quantity of solid in the volume $v$ of solvent. When no heat or other energy is received from outside sources, the heat, whether work be done or not, will be taken from the solvent. If the operation be conducted isothermally, heat will be taken from the surroundings whether mechanical work be done or not. But the fact that work may be done and transformed into heat while the volume changes from $v_{1}$ to $v_{2}$, compels us to conclude that in either case, whether work has been done or not, since initial and final states are the same if conducted isothermally, then if it be possible by any mechanical means whatever to restore the solution to its original state after dilution, the mechanical work necessary must be greater, or in the limit equal to, the maximum work possible to be obtained by dilution. To suppose that less than this minimum be sufficient, is equivalent to supposing that unlimited work can be done by means of the heat of the surroundings. This possibility is denied by the second law of thermodynamics. Therefore, if $\mathrm{W}$ is the maximum work possible to be obtained by diluting a solution from the volme $v_{1}$ to volume $v_{2}$ and the process of restoration be one of filtration through any septum, the minimum work required cannot be less than

$$
\mathrm{W}=\int_{v_{3}}^{v_{1}} \mathrm{P} d v=-\int_{v_{2}}^{v_{2}} p d v,
$$

where $P$ is the minimum filtration pressure necessary and $d v$ the increment of volume swept through. During a state of equilibrium in the absence of mechanical motion, $\mathrm{P}=-p$ since the two pressures are oppositely directed. Here no 
hypothesis need to be offered to account for the resistance to filtration, although the mind may eagerly seek an explanation. This filtration pressure answers all the requirements necessary to satisfy the equation

$$
\mathrm{W}=\rho g h v \log \frac{v_{1}}{v_{2}} .
$$

It is entirely independent of any hypothesis as to the constitution of matter and of any phenomena of osmosis except those arising from interacting vapors and liquids, and might have been deduced had Pfeffer's experiment never been observed. Professor Ostwald refers to an experiment, ${ }^{1}$ without, however, giving any details in which the filtration pressure above referred to is demonstrated experimentally. This, I take it, is the fundamental fact which may be deduced from general dynamic principles and demonstrated experimentally. Since it is entirely independent of any hypothesis it furnishes a rational dynamic basis for a theory of solutions. From the fundamental equation $\mathrm{W}=\int \mathrm{P} d v$ we obtain at once a rational dynamic definition of filtration pressure above referred to in the form $\mathrm{P}=\frac{d \mathrm{~W}}{d v}$ or the work per unit volume of solvent which must be done in separating solute and solvent. Osmotic pressure is, then, dynamically considered, the hydrostatic pressure arising through osmosis which just balances the filtration pressure just defined. It is evident that pressure arising from the osmotic process cannot exceed this limiting filtration value which, if exceeded, will reverse the process that produced it. This limiting value has been used under the name of osmotic pressure and has consequently given consistent dynamic results; but physically, osmotic pressure has no significance or existence except as the hydrostatic pressure produced by osmosis through semi-permeable septa. The fanciful idea that there is an occult kind of osmotic pressure

${ }^{1}$ Ostwald: Principles of Inorganic Chemistry. Translated by Findlay, p. 650. 
"that would break the glass if it reached it," arises from an overstrained analogy.

There is, however, yet another aspect to this value of $\mathrm{P}$, in the equation $p v=\mathrm{PV}$. This equation was derived from considerations of static equilibrium based on the kinetic equilibrium between the active masses of solvent whether liquid or vapor. From it we should be able to obtain the "intrinsic pressure," as it is called, of the liquid solvent. Two cases by two different methods will suffice.

Let us take $1 \mathrm{gm}$ of water at $0^{\circ} \mathrm{C}$. From tables we find that its pressure in the vapor state is $\frac{46}{76}$ atm. and its volume 204 liters, or $204000 \mathrm{cc}$. Since $\mathrm{V}$-in the liquid state is $\mathrm{I} c c$ we have $\mathrm{P}=p v=\frac{.46}{76} \times 204000=\mathrm{I} 235$ atmospheres. This does not mean that there is, during equilibrium, such a pressure actually existing, but that under certain circumstances such a force would have to be reckoned with. In terms of molecules, it means merely that in the liquid state, at that temperature, we have 1235 times as many molecules acting through a given element of area as we find in the vapor state. In terms of mass-action there is I 235 times as much mass concerned in the liquid state as compared with the vapor state under standard conditions.

This value, I 200 atmospheres, for the intrinsic pressure of water at $o^{\circ} \mathrm{C}$, may be corroborated by substituting experimental values in the equation expressing the conditions of equilibrium between vapor and liquid and liquid solution,

$$
\frac{p_{1}-p_{2}}{p_{1}}=\frac{P_{1}-P_{2}}{P_{1}}
$$

In Sentner's Physical Chemistry, ${ }^{1}$ p. I05, is quoted an experiment from Smits where the following numerical values are given for an aqueous sugar solution at $0^{\circ} \mathrm{C}$ :

$$
\frac{0.00705}{4.62}=\frac{29.0358 \times 18}{1000 \times 34^{2}} \text {. }
$$

${ }^{1}$ Outlines of Physical Chemistry, G. Sentner, r9o8. 
Here $p_{1}=4.62$ and $p_{1}-p_{2}=0.00705$. Calculating $\mathrm{P}_{1}-\mathrm{P}_{2}$, the osmotic pressure, from the above data, we get $\mathrm{P}_{1}-\mathrm{P}_{2}=$ I.9 atmospheres. Therefore $P_{1}$, the intrinsic pressure, $=$ I240 atm.

An example ready at hand taken from Nernst ${ }^{1}$ will illustrate the influence of molecular weight on intrinsic pressure of a liquid in the case of benzene at $80^{\circ} \mathrm{C}$. Here $p_{1}-p_{2}=$ 9.26, $p_{1}=75 \mathrm{I} .86$. In like manner, as above, we find the osmotic pressure $\mathrm{P}_{1}-\mathrm{P}_{2}$ of benzene at $80^{\circ} \mathrm{C}$ under the given conditions to be 3.75 atmospheres (Nernst gets 3.78 instead of 3.75 through using $p_{2}$ for $p_{1}$ ). By substitution we get.

$$
\frac{9.26}{75 \mathrm{I} .86}=\frac{3.75}{\mathrm{P}_{1}} \text {. }
$$

where $\mathrm{P}_{1}$ is the intrinsic pressure of benzene at $80^{\circ} \mathrm{C} . \because \mathrm{P}_{1}=$ 304 atmospheres.

\section{Summary and Conclusion}

Attention has been called to certain inconsistencies between the experimental facts and the nomenclature arising from the theory that osmotic pressure is a direct function, or property, of the solute. I have shown how a number of fundamental facts may be interpreted without reference to osmosis at all,--merely as activities of the solvent brought into play by the presence of a foreign substance. In addition, I have shown how a new and important set of facts is brought to light by the application of the principle of massaction to deriving an equation of equilibrium between the vapor and liquid states having the simple form

$$
\frac{p_{1}-p_{2}}{p_{1}}=\frac{\mathrm{P}_{1}-\mathrm{P}_{2}}{\mathrm{P}_{1}}
$$

This equation affords a simple means of determining the intrinsic pressure of a liquid by comparatively simple and direct experimental measurements. It is more fundamental than the general gas (van't Hoff's) equation for that can

${ }^{1}$ Nernst: Theoretical Chemistry, $4^{\text {th }}$ ed. Translated by Lehfeldt, p. $x_{3} 8$. 
readily be derived from it as shown in the text. By a direct measurement of vapor pressure and osmotic (or filtration) pressure, without reference to the number of molecules concerned, the intrinsic pressure of a liquid solvent may be determined.

In the light of all the evidence adduced, can there be longer any reasonable doubt that the present theory of osmotic pressure is open to revision?

State College, Pa., Jan. IO, I9IO 